\title{
Plasmonic Nanostructures for Energy Application
}

\author{
Nilesh Kumar Pathak* \\ Department of Physics, Maharaja Agrasen College, University of Delhi, New Delhi, India
}

The present work furnishes the optical properties of metal nanostructures and their application in energy. Metal supports plasmonic resonances that can be tuned in the desired range of the solar spectrum by changing its size, shape, and surrounding environment. Two different systems, isolated and coupled gold metal nanospheres, are taken into account to study optical properties. These optical properties of the chosen nanostructure are analyzed in terms of surface plasmon resonances using the Boundary Element Method (BEM). The tuning of surface plasmon resonances under different parameter domains and their applications in energy are the main thrusts of this work. The simulated extinction spectrum and corresponding spectral width of metallic nanogeometries could be used in several applications like solar stills, solar collectors, and solar cells.

\section{OPEN ACCESS}

Edited by:

Mariana Amorim Fraga,

Federal University of São Paulo, Brazil

Reviewed by:

Diego Alexandre Duarte,

Federal University of Santa

Catarina, Brazil

Daniel Neves Micha,

Federal Center for Technological

Education Celso Suckow da

Fonseca, Brazil

*Correspondence:

Nilesh Kumar Pathak

nileshpiitd@gmail.com

Specialty section:

This article was submitted to

Micro- and Nanoelectromechanical

Systems,

a section of the journal

Frontiers in Mechanical Engineering

Received: 11 April 2020

Accepted: 09 June 2020

Published: 21 August 2020

Citation:

Pathak NK (2020) Plasmonic

Nanostructures for Energy

Application. Front. Mech. Eng. 6:53.

doi: 10.3389/fmech.2020.00053
Keywords: plasmon, nanostructure, SPR, BEM, extinction

\section{INTRODUCTION}

Solar energy is the utmost abundantly available, clean, pollution-free, and economically viable energy source on the Earth, and the sun is the ultimate source of this solar energy. The sun will supply an unlimited amount of energy to the Earth for an unlimited time due to the abundance of hydrogen in its core. This abundance of hydrogen inside the sun's core fuses together to produce energy known as solar energy. The sun is continuously supplying solar energy without any expectation therefore, everyone who is working in this field wants to use this freely available energy (Aybar, 2006; Govorov and Richardson, 2007; Modi and Nayi, 2020). Solar energy attracts the scientific community because it is economically viable, free from pollution, and available throughout the year. Due to its inherent features, it is believed that solar energy is an alternative source of energy (Atwater and Polman, 2010; Liu et al., 2011; Pathak et al., 2014). There are a variety of technologies available throughout the globe that are being used to harness sunlight. These technologies include solar photovoltaics, solar stills, solar cookers, and solar heat collectors. In solar photovoltaics, solar energy is converted into electrical energy by using a solar cell device. A solar still is a kind of technology that is used to obtain fresh water from seawater/brackish water (Duffie and Beckman, 1991; Shukla and Modi, 2017). A solar cooker is used for cooking food by solar radiation, and a solar heat collector is used to collect the sun's radiation for distilling and heating water for kitchen and general home purposes (Riffat and Cuce, 2011; Cuce and Cuce, 2013; Modi and Modi, 2019). Several efforts have been made in the last three to four decades to design, simulate, and fabricate a variety of technologies to harness sunlight. Researchers are still working on these technologies to optimize the various parameters for better output efficiency (Park, 2015; Pathak et al., 2017).

There have been several upgrades to optimize the parameters of the said technologies for better output. The upgrades have been in terms of the miniaturization of technologies. Miniaturization means shifting the technologies from a larger scale to the nanoscale domain. In the nanoscale 
domain, the optical and electrical responses of materials change dramatically. These nanoscale responses of materials are utilized by research communities and industries. The key objective of all of the above-mentioned technologies is to trap more sunlight by absorption. For example, (1) in a solar cell, the higher the photon absorption inside the active material, the more electricity will be generated, and (2) in a solar still, the efficiency of the still can be enhanced by heating/vaporization of water by sunlight. At present, there is sufficient available literature discussing the optimal device design, choice of materials, and material structures for achieving better efficiency (Duffie and Beckman, 1991; Riffat and Cuce, 2011; Shukla and Modi, 2017; Modi and Modi, 2019; Modi and Nayi, 2020). However, in order to resolve the energy problem, researchers are still trying to develop innovative technologies and devices that reduce the use of conventional sources of energy. Since the Earth receives nearly 3.85 million EJ (Exa-Joule) of solar energy every year, we are motivated to think about this freely available energy and how it could beneficial for society. In order to improve the efficiency of the above-said technologies, many researchers have used the concept of plasmonic metal nanostructures in different ways for different purposes. With this nascent technology, one can increase the light-trapping capability for one's own purpose. For example, metal nanoparticles can be used in a solar still as nanofluids and will improve the output efficiency. The metal nanoparticles present in these nanofluids absorb the sunlight and produce heat. Meanwhile, in a solar cell, metal nanoparticles have been scattered onto the surface of active materials (materials that are responsible for producing current) with different concentrations and coverage factors. Under the influence of solar radiation, these metal nanoparticles improve the photon absorption capability of solar cell material (Ji et al., 2015; Pathak et al., 2016; Sharshir et al., 2017; Modi et al., 2019, 2020).

Plasmonics is a very recent and new branch of nanophotonics that has been developed over the last two decades due to its potential application in every field of science and technology. It focuses on phenomena related to the plasmon, which is a quantum of plasma oscillation like a photon, phonon, exciton, or magnon. The plasmon that generally appears in metal nanostructures is the collective oscillation of electrons, which oscillate at a certain frequency known as the plasmon frequency (Kelly et al., 2003; Le et al., 2005; Pathak and Sharma, 2016; Wang et al., 2017). When the metal nanostructures are irradiated with an incident electromagnetic field in a range of frequency matching with metal's plasmon frequency, a resonance known as

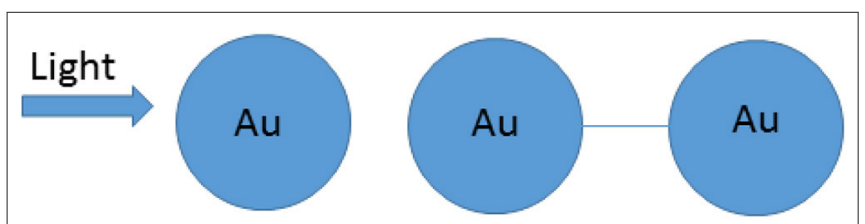

FIGURE 1 | Interaction of light with an isolated nanosphere and coupled nanospheres surrounded by water. surface plasmon resonance (SPR) is established (Esteban et al., 2012; Pandey et al., 2016, 2017). The main feature of SPR is that it can be tuned in a desired range of the solar spectrum by choosing different types of metal nanostructures of different size and shape. The plasmon resonances exhibited by metal nanostructures are visualized in terms of scattering, absorption, and extinction spectrum. The main thrust of this work is to study the extinction spectrum of gold metal nanostructures in two different cases that cover the regime of the solar spectrum.

\section{MODELING OF NANOSTRUCTURES}

Simulation of the optical response of gold nanostructures was performed by using the metal nanoparticle boundary element method (MNPBEM) Matlab-based toolbox. This is a wellknown toolbox, and its details can be found in the literature (Hohenester and Trügler, 2012). The main purpose of this toolbox is to solve Maxwell's equations for different types of metal nanostructures surrounded by homogeneous dielectric environments. The MNPBEM toolbox has the advantage over the other techniques that it takes less computation time to produce an output. It discretizes the boundary between the different dielectric environments instead of the whole volume, as in the case of the DDA (Discrete Dipole Approximation) and FDTD (Finite Difference Time Domain) methods. In this article, isolated and coupled gold nanospheres are taken into account, and their optical properties are simulated in terms of the extinction spectrum. The embedding media is water, which has a constant refractive index (1.32), and the dielectric constant of gold has been taken from the literature (Palik, 1985; Draine and Flatau, 2012; Pathak et al., 2019). Here, our interest is in calculating the optical cross-sections of metal nanostructure when it is irradiated with light using the Boundary Element Method. The optical cross-sections are expressed in terms of the Poynting vector, the details of which are taken from the literature (Bohren and Huffman, 1998; Jackson, 1999). The optical cross-sections in terms of a field are expressed as

$$
\begin{aligned}
C_{s c a} & =n_{b} \int_{\partial \Omega}(\hat{n} \cdot(E \times B)) R d s \\
C_{e x t} & =\frac{1}{n_{b}} \int_{\partial \Omega}\left(\hat{n} \cdot\left(E \times B_{0}^{*}+E_{0}^{*} \times B\right)\right) R d s \\
C_{a b s} & =C_{\text {ext }}-C_{s c a}
\end{aligned}
$$

where $n_{b}$ is the refractive index and $\hat{n}$ a normal vector to the surface $d s, \mathrm{R}$ is the radius vector, and $\partial \Omega$ is the volume element. $\mathrm{E} \times \mathrm{B}$ is the Poynting vector, which is taken from the literature (Bohren and Huffman, 1998). The nanostructure model is shown in Figure 1.

\section{RESULTS AND DISCUSSION Isolated Spherical Gold Nanostructure}

The interaction of solar light radiation with the metal nanostructures is studied in terms of the extinction crosssections. These are the cross-sections under the influence of radiation and are different from geometrical cross-sections of the 

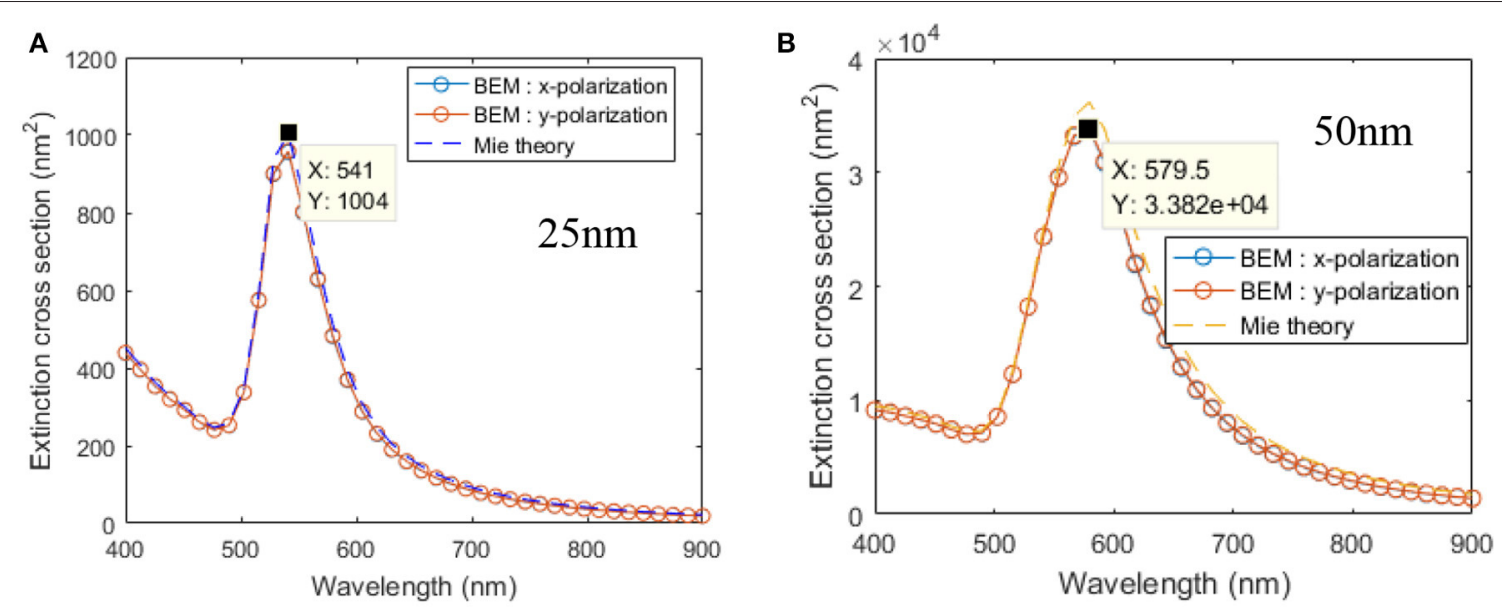

FIGURE 2 | Extinction cross-section of (A) a 25-nm- and (B) a 50-nm-radius gold nanosphere surrounded by water.

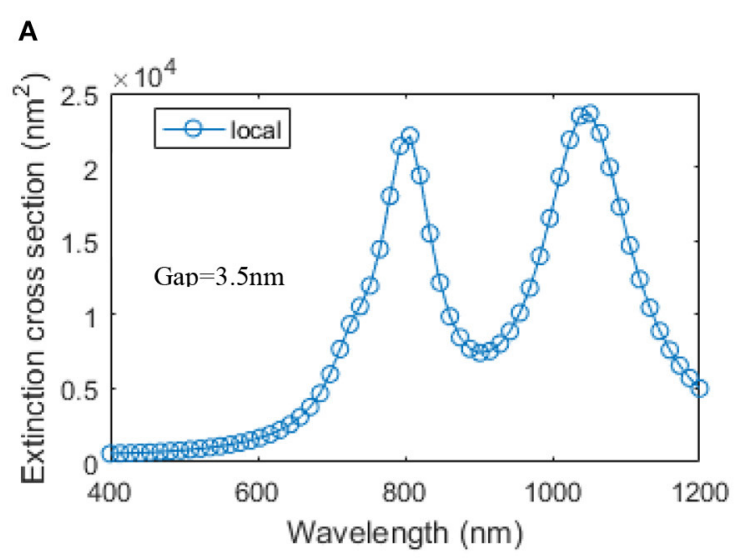

B

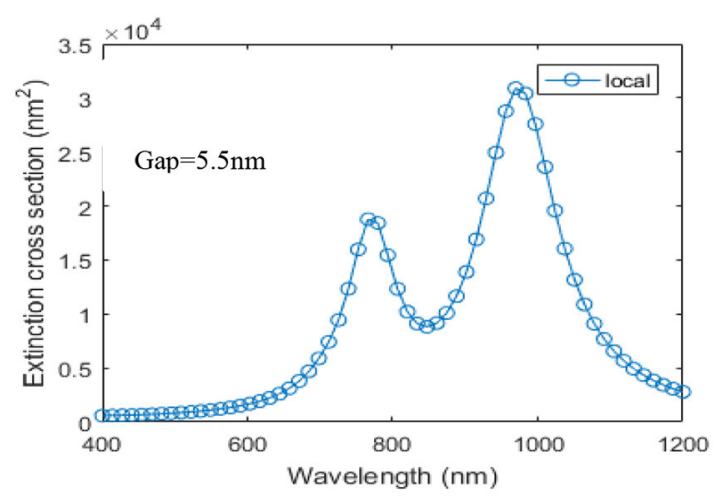

FIGURE 3 | Extinction cross-section of 25-nm-radius coupled gold nanospheres separated by gaps of (A) $3.5 \mathrm{~nm}$ and (B) $5.5 \mathrm{~nm}$ surrounded by water.

nanostructure. When light incident upon the nanostructure is absorbed, radiation creates a virtual region near the surface that is very hot due to the absorption of light. The influence of the hot zone is not restricted in the particle volume: it spreads to a certain distance near the surface of the particle. The observed hot zone near the surface of a metal nanostructure is the main object of interest for researchers. For example, it could be utilized for several purposes like water heating and evaporation. The extinction spectra of spherical gold nanostructures with two different diameters were analyzed and are shown in Figure 2. The purpose of plotting the extinction spectrum against the wavelength is that it clearly indicates light-metal interaction in a particular band of the solar spectrum. This band of the solar spectrum can be tuned in the desired range by changing various parameters like the size and shape of the nanostructure and the medium in which it is embedded.

Two different polarizations of lightwaves were used to irradiate the gold nanosphere, and the results obtained were compared with those from the Mie theory. Mie theory is the exact solution of Maxwell's equation that is valid for nanostructures with arbitrary size and spherical shape. From the figure, it can be observed that BEM and Mie theory are in good agreement for this situation. The surface plasmon resonance (SPR) peak of the 25-nm-radius gold nanosphere surrounded by water $(n=$ 1.32) lies at $541 \mathrm{~nm}$, while that of the 50 -nm-radius nanosphere is at $579 \mathrm{~nm}$. The spectral width in the 25-nm-radius case is smaller than that for the 50 -nm-radius sphere. A greater width of the SPR resonance clearly suggests more interaction between the solar spectrum and the nanostructure. This interaction means that the chosen nanostructure harvests more solar light in the stated wavelength range. This interaction concept in terms of the absorption/extinction spectrum could be utilized for heating the nanofluids used in a solar still.

\section{Coupled Spherical Gold Nanostructure}

The extinction spectrum of a coupled spherical gold nanostructure separated by a certain gap is explored in Figure 3. For a single sphere, as discussed above, a single SPR peak with a 
certain spectral width was observed, while in a coupled system, dual SPR peaks are observed. This is the main advantage of a coupled nanostructure over the single/isolated nanosphere. For the case of 25-nm-radius nanospheres separated by a distance of $3.5 \mathrm{~nm}$, SPR peaks are exhibited in two different regimes of the solar spectrum, one at $800 \mathrm{~nm}$ and other at $1,050 \mathrm{~nm}$. In this coupled spherical system, the radius of each sphere is $25 \mathrm{~nm}$, and they are separated by gaps of 3.5 or $5.5 \mathrm{~nm}$. Both of the SPR peaks corresponding to the $3.5-\mathrm{nm}$ gap have almost equal extinction magnitude.

As the separation gap between the two gold sphere increases from 3.5 to $5.5 \mathrm{~nm}$ SPR peaks are blue-shifted and develop different extinction magnitudes, as shown in Figure 3B. This shifting of SPR peaks in a wide range of the solar spectrum can be controlled by controlling the gap parameter in the coupled system. For the coupled nanospheres, it was also observed that the SPR peaks lie in the infrared region of the solar spectrum. Therefore, in the coupled system, by tuning the gap parameter, one can have both the features of an isolated nanosphere and coupled nanospheres. The dual SPR peaks of the surface plasmon will affect the device twice at a time, which is an additional advantage of coupled spheres. If this structure were to be used in the design of gold nanoparticle nanofluids in a solar still, the fluid would be heated up more in this spectral wavelength range.

\section{CONCLUSION}

The present work focuses on the study of the extinction spectrum of isolated as well as coupled gold nanospheres. The optimized parameters regarding size, shape, and separation gap are explored using MNPBEM toolbox. The simulated extinction spectra of isolated and coupled gold nanospheres surrounded by water

\section{REFERENCES}

Atwater, H. A., and Polman, A. (2010). Plasmonics for improved photovoltaic devices. Nat. Mater. 9, 205-213. doi: 10.1038/nmat2629

Aybar, H. S. (2006). Mathematical modeling of an inclined solar water distillation system. Desalination 190, 63-70 doi: 10.1016/j.desal.2005.07.015

Bohren, C. F., and Huffman, D. R. (1998). Absorption and Scattering of Light by Small Particles. (New York, NY: Wiley).

Cuce, E., and Cuce, P. M. (2013). A comprehensive review on solar cookers. Appl. Energ. 102, 1399-1421. doi: 10.1016/j.apenergy.2012.09.002

Draine, B. T., and Flatau, P. J. (2012). User Guide to the Discrete Dipole Approximation Code DDSCAT 7.2. Available online at: http://arxiv.org/abs/ 1202.3424 (accessed August 25, 2020).

Duffie, J. A., and Beckman, W. A. (1991). Solar Engineering of Thermal Processes. (New York, NY: Wiley).

Esteban, R., Borisov, A. G., Nordlander, P., and Aizpurua, J. (2012). Bridging quantum and classical plasmonics with a quantum-corrected model. Nat. Commun. 3:825. doi: 10.1038/ncomms1806

Govorov, A. O., and Richardson, H. H. (2007). Generating heat with metal nanoparticles. Nano Today 2, 30-38. doi: 10.1016/S1748-0132(07)70017-8

Hohenester, U., and Trügler, A. (2012). MNPBEM - A Matlab toolbox for the simulation of plasmonic nanoparticles. Comp. Phys. Commun.183, 370-381 doi: 10.1016/j.cpc.2011.09.009

Jackson, J. D. (1999). Classical Electrodynamics. (New York, NY: Wiley).

Ji, A., Sharma, R., Pathak, H., Pathak, N. K., and Sharma, R. P. (2015). Numerical simulation of plasmonic light trapping in thin-film $\mathrm{Si}$ solar cover a broad range of the solar spectrum ranging from the visible to the infrared. The simulation work simply suggests that the scientific energy community should take other metals that can be coated with non-metallic materials and study their extinction or absorption behavior in a solar energy harvesting context. Special emphasis has been given to the gap parameter, which is the distance between the nanospheres. It is concluded that, for the given size, shape, and material, a separation of $3.5 \mathrm{~nm}$ is optimal in this simulation. This study also opens up the new idea that researchers should simulate or fabricate a variety of nanostructures and study their extinction spectra for several applications. The model presented is restricted to single and coupled nanospheres, but it can be further extended to study the extinction spectrum of coupled nanospheroids, coupled nanorods, and chains of nanoparticles.

\section{DATA AVAILABILITY STATEMENT}

The raw data supporting the conclusions of this article will be made available by the authors, without undue reservation.

\section{AUTHOR CONTRIBUTIONS}

NP prepared the whole manuscript in terms of the idea and the simulation.

\section{ACKNOWLEDGMENTS}

The author would like to thank the Principal at Maharaja Agrasen College, University of Delhi, Delhi-110096, India, for his support. The author would also like to thank Prof. R P Sharma, CES IIT Delhi, India, for his motivational support. cells: surface coverage effect. J. Phys. D: Appl. Phys. 48, 275101-275107. doi: $10.1088 / 0022-3727 / 48 / 27 / 275101$

Kelly, K. L., Coronado, E., Zhao, L. L., and Schatz, G. C. (2003). The optical properties of metal nanoparticles: the influence of size, shape, dielectric environment. J. Phys. Chem. B 107, 668-677. doi: 10.1021/jp026731y

Le, F., Lwin, N. Z., Steele, J. M., Kall, M., Halas, N. J., and Nordlander, P. (2005). Plasmons in the metallic nanoparticle/film system as a tunable impurity problem. Nano Lett. 5, 2009-2013. doi: 10.1021/nl0515100

Liu, Z. W., Hou, W. B., Pavaskar, P., Aykol, M., and Cronin, S. B. (2011). Plasmon resonant enhancement of photocatalytic water splitting under visible illumination. Nano Lett. 11, 1111-1116. doi: 10.1021/nl104005n

Modi, K. V., Jani, H. K., and Gamit, I. D. (2020). Impact of orientation and water depth on productivity of single-basin dual-slope solar still with $\mathrm{Al} 2 \mathrm{O} 3$ and $\mathrm{CuO}$ nanoparticles. J. Therm. Anal. Calorim. 137, 1279-1294. doi: 10.1007/s10973-020-09351-1

Modi, K. V., and Modi, J. G. (2019). Performance of single-slope double-basin solar stills with small pile of wick materials. Appl. Therm. Eng. 149 723-730. doi: 10.1016/j.applthermaleng.2018.12.071

Modi, K. V., and Nayi, K. H. (2020). Efficacy of forced condensation and forced evaporation with thermal energy storage material on square pyramid solar still. Renewab. Energy. 153, 1307-1319. doi: 10.1016/j.renene.2020.02.095

Modi, K. V., Shukla, D. L., and Ankoliya, D. B. (2019). A comparative performance study of double basin single slope solar still with and without using nanoparticles. J. Sol. Energy Eng. Trans. ASME 141:3. doi: 10.1115/1.4041838

Palik, E. D. (1985). Handbook of Optical Constants of Solids. (Orlando, FL: Academic). 
Pandey, G. K., Pathak, N. K., Ji, A., Uma, R., and Sharma, R. P. (2016). Study of surface enhanced raman scattering of single molecule adsorbed on the surface of metal nanogeometries: electrostatic approach. Plasmonics 11, 1343-1349. doi: 10.1007/s11468-016-0181-x

Pandey, G. K., Pathak, N. K., Uma, R., and Sharma, R. P. (2017). Electromagnetic study of surface enhanced raman scattering of plasmonic-biomolecule: an interaction between nanodimer and single biomolecule. Solid State Commun. 255, 47-53. doi: 10.1016/j.ssc.2017.03.010

Park, N. G. (2015). Perovskite solar cells: an emerging photovoltaic technology. Mater. Today 18, 65-72. doi: 10.1016/j.mattod.2014.07.007

Pathak, N. K., Chander, N., Komarala, V. K., and Sharma, R. P. (2017). Plasmonic perovskite solar cells utilizing Au@SiO2 core-shell nanoparticles. Plasmonic 12, 237-244. doi: 10.1007/s11468-016-0255-9

Pathak, N. K., Ji, A., and Sharma, R. P. (2014). Study of efficiency enhancement in layered geometry of excitonic plasmonic solar cell. App. Phys. A 115, 1445-1450. doi: 10.1007/s00339-013-8061-0

Pathak, N. K., Parthasarathi, Kumar, P. S., and Sharma, R. P. (2019). Tuning of the surface plasmon resonance of aluminum nanoshell near-infrared regimes, Phys. Chem. Chem. Phys. 21, 9441-9449. doi: 10.1039/C9CP $01115 \mathrm{C}$

Pathak, N. K., Pathak, H., Pandey, G. K., Ji, A., and Sharma, R. P. (2016). Study of external quantum efficiency of plasmonic coupled bilayer active device: influence of layer thickness and nanoparticle filling factor. Appl. Phys. A 122, 1048-1056. doi: 10.1007/s00339-016-0574-x

Pathak, N. K., and Sharma, R. P. (2016). Study of broadband tunable properties of surface plasmon resonances of noble metal nanoparticles using mie scattering theory: plasmonic perovskite interaction. Plasmonics 11, 713-719. doi: 10.1007/s11468-015-0097-x

Riffat, S. B., and Cuce, E. (2011). A review on hybrid photovoltaic/thermal collectors and systems. Int. J. Low-Carbon Tech. 6, 212-241. doi: $10.1093 /$ ijlct/ctr016

Sharshir, S. W., Peng, G., Wu, L., Yang, N., Essa, F. A., Elsheikh, A. H., et al. (2017). Enhancing the solar still performance using nanofluids and glass cover cooling: experimental study. Appl. Therm. Eng. 113, 684-693. doi: 10.1016/j.applthermaleng.2016.11.085

Shukla, D. L., and Modi, K. V. (2017). A technical review on regeneration of liquid desiccant using solar energy. Renew. Sustain. Energy Rev. 78 517-529. doi: 10.1016/j.rser.2017.04.103

Wang, H., Wang, X., Yan, C., Zhao, H., Zhang, J., Martin, O. J., et al. (2017) Full color generation using silver tandem nanodisks. Nano Lett. 17, 2265-2272. doi: 10.1021/acsnano.6b08465

Conflict of Interest: The author declares that the research was conducted in the absence of any commercial or financial relationships that could be construed as a potential conflict of interest.

Copyright (c) 2020 Pathak. This is an open-access article distributed under the terms of the Creative Commons Attribution License (CC BY). The use, distribution or reproduction in other forums is permitted, provided the original author(s) and the copyright owner(s) are credited and that the original publication in this journal is cited, in accordance with accepted academic practice. No use, distribution or reproduction is permitted which does not comply with these terms. 